\begin{tabular}{|c|c|c|c|c|c|c|c|c|}
\hline & \multicolumn{2}{|c|}{ Size } & \multicolumn{2}{|c|}{ Resection type } & \multicolumn{2}{|c|}{ Site } & \multicolumn{2}{|c|}{ Scarred } \\
\hline & $\leq 50 \mathrm{~mm}$ & $>50 \mathrm{~mm}$ & En bloc & P meal & LC & $\mathrm{RC}$ & yes & no \\
\hline \multirow[t]{2}{*}{ All polyp recurrence $14 / 106(13 \%)$} & $6 / 78(7.6 \%)$ & $8 / 28(28.5 \%)$ & $1 / 42(2.3 \%)$ & $7 / 44(15.9 \%)$ & $13 / 83(15.6 \%)$ & $1 / 23(4.3 \%)$ & $6 / 20(30 \%)$ & $8 / 86(9.3 \%)$ \\
\hline & \multicolumn{2}{|c|}{$P=0.009$} & \multicolumn{2}{|c|}{$P=0.001$} & \multicolumn{2}{|c|}{$P=0.15$} & \multicolumn{2}{|c|}{$P=0.024$} \\
\hline \multirow[t]{2}{*}{ Unscarred polyp recurrence $8 / 86(9.3 \%)$} & $2 / 62(3.2 \%)$ & $6 / 24(25 \%)$ & $1 / 42(2.3 \%)$ & $7 / 44(15.9 \%)$ & $8 / 68(11.7 \%)$ & 0/18 (0\%) & & \\
\hline & \multicolumn{2}{|c|}{$P=0.005$} & \multicolumn{2}{|c|}{$P=0.058$} & \multicolumn{2}{|c|}{$P=0.195$} & & \\
\hline
\end{tabular}

This is followed by variable degrees of SM dissection and completion of circumferential mucosal incision. Finally a snare assisted resection is performed either en bloc or piecemeal, depending on the polyp size and extent of SM dissection.

Results 127 polyps in 127 patients of mean age 71 years. Mean polyp size $46 \mathrm{~mm}(20-170 \mathrm{~mm}) .27 \%$ were $>50 \mathrm{~mm}$. $27 \%$ were scarred from past attempted resection. $26 \%$ were in the right colon.

En bloc resection: $58 / 127(46 \%)$. Size $<50 \mathrm{~mm}$ was a significant $(\mathrm{p}=0.001)$ predictor of en bloc resection ( 88 vs. $12 \%)$.

The complication rate was $11 / 127(8.6 \%)$ with $5(3.9 \%)$ bleeds, 4(3.1\%) diathermy damage to muscle fibres and 1 $(0.78 \%)$ perforation. Complications were not linked to polyp size, scarring or resection site. A single patient with perforation required surgery. All other complications were managed endoscopically.

The recurrence rate was $14 / 106(13 \%)$. This was significantly higher for polyps $>50 \mathrm{~mm}(\mathrm{p}=0.009)$ and in scarred polyps $(\mathrm{p}$ $=0.024$ ).

On sub-analysis of unscarred polyps, polyps $\leq 50 \mathrm{~mm}$ with no scarring had a very low recurrence rate of $3.2 \%$ as compared to $25 \%$ in polyps $>50 \mathrm{~mm}(\mathrm{p}=0.005)$.

Factors associated with recurrence

Conclusion This is the largest reported western series demonstrating the feasibility, safety and efficacy of KAR for large and refractory polyps, with or without scarring, at all colonic sites. Our data demonstrates that complications of KAR are not related to size but the recurrence rate is. Size $>50 \mathrm{~mm}$ and scarring seem to be predictors of recurrence.

We propose flat polyps $20-50 \mathrm{~mm}$ in size as the ideal indication for KAR in the western setting.

Disclosure of Interest None Declared.

\section{OC-014 PATIENTS WITH INTRAMUCOSAL CARCINOMA ARISING IN BARRETT'S EOSOPHAGUS HAVE SIMILAR OUTCOMES TO THOSE WITH HIGH GRADE DYSPLASIA: DATA FROM THE UNITED KINGDOM REGISTRY}

${ }^{1,2}{ }^{2} \mathrm{RJ}$ Haidry ${ }^{*},{ }^{1} \mathrm{M}$ Banks, ${ }^{1} \mathrm{~A}$ Gupta, ${ }^{2} \mathrm{M}$ Butt, ${ }^{3} \mathrm{G}$ Fullarton, ${ }^{4} \mathrm{H}$ Smart, ${ }^{3} \mathrm{~J}$ Morris, ${ }^{5} \mathrm{R}$ Willert, ${ }^{6} \mathrm{R}$ Narayanasamy, ${ }^{1} \mathrm{M}$ Rodriguez-Justo, ${ }^{1} \mathrm{M}$ Novelli, ${ }^{1,2} \mathrm{~L}$ Lovat. ${ }^{1} U C L H$, London, UK; ${ }^{2} N M L C$, UCL, London, UK; ${ }^{3} G R I$, Glasgow, UK; ${ }^{4} R L U H$, Liverpool, UK; ${ }^{5} M R I$, Manchester, UK: ${ }^{6}$ St James Hospital, Dublin, Ireland

\subsection{6/gutinl-2014-307263.14}

Introduction Mucosal neoplasia arising in Barrett's oesophagus (BE) can be treated with combined radiofrequency ablation (RFA) and endoscopic mucosal resection (EMR). Once there is submucosal invasion the risk of lymph node metastases increases and surgery is favoured. High grade dysplasia (HGD) and intramucosal carcinoma (IMC) arising from the mucosal layer in BE are defined as separate entities in the revised Vienna classification (category 4.1 and 4.4 respectively).

Methods We examine prospective data from United Kingdom (UK) registry of patients undergoing RFA/EMR for BE neoplasia over past 5 years to compare outcomes between HGD and IMC patient cohorts. Histological confirmation of HGD or IMC was required on two separate occasions by specialist histopathologists prior to treatment. Before RFA, visible lesions were removed by EMR. Thereafter patients underwent RFA 3 monthly following which biopsies were taken at 12 months for clearance of dysplasia (CR-D) and BE (CR-IM). Twelve month outcomes, frequency of EMR, cancer progression and long term durability in both groups were examined.

Results To date 367 patients with HGD and 125 with IMC have been treated. There is no difference in age, sex, baseline BE length between the 2 groups. EMR prior to RFA is far more prevalent in IMC cohort than HGD patients (78 vs. 45\%, P < 0.0001). Patients had an average of 2-3 RFA treatments over 12 months (range 1-6) in both cohorts. Rescue EMR after starting RFA for new lesions was similar in both groups (HGD 7\%, IMC 6.5\%). CR-D and CR-IM in the HGD cohort was 85 and 69\% respectively at 12 months. This was not significantly different in the IMC cohort ( 86 and $71 \%, \mathrm{p}=0.7$ ). Overall progression to invasive cancer was not significantly different in either cohort (HGD 4.1\%, IMC 7.2\%). Kaplan Meir survival statistics did not show any difference in long term durability of successful neoplasia treatment in both groups $(\mathrm{p}=0.9, \log$ rank test), median follow up 20 months.

Conclusion We report one of the largest series of patients undergoing endoscopic therapy for IMC arising in BE. Patients with IMC are more likely to have visible lesions that require EMR prior to RFA than those with HGD. However, once all visible lesions are removed, there is no statistical difference in clinical outcomes between the cohorts. Minimally invasive endoscopic therapy with RFA/EMR is a safe and effective treatment in patients with IMC. All collaborators of the UK RFA registry are acknowledged for their contributions to this work

Disclosure of Interest None Declared.

\section{Joint oesophageal and gastroduodenal free papers}

\section{OC-015 HIGH GRADE DYSPLASIA ARISING IN BARRETT'S OESOPHAGUS CAN BE ACCURATELY DIAGNOSED COMBINING FOURIER TRANSFORM INFRARED SPECTROSCOPY AND SINGLE ELEMENT ATTENUATED TOTAL REFLECTANCE FTIR DATA}

${ }^{1} \mathrm{~L}$ Foreman, ${ }^{2,3} \mathrm{RJ}$ Haidry*, ${ }^{2} \mathrm{M}$ Banks, ${ }^{2} \mathrm{M}$ Novelli, ${ }^{1} \mathrm{M}$ Rodriguez-Justo, ${ }^{4} \mathrm{~J}$ Dougan, ${ }^{4} S$ Kazarian, ${ }^{1} \mathrm{P}$ Rich, ${ }^{2,3} \mathrm{~L}$ Lovat. ${ }^{1} U C L$, London, UK; ${ }^{2} U C L H$, London, UK; ${ }^{3} N M L C, U C L$, London, UK; ${ }^{4}$ mperial College, London, UK

\subsection{6/gutjnl-2014-307263.15}

Introduction Fourier transform infrared spectroscopy (FTIR) detects specific molecules in human tissue from unique vibrational absorption spectra in the mid Infra-Red region (1800- 